\title{
Effect of the interatomic potential stiffness on the properties of gap discrete breathers in 2D biatomic Morse crystal
}

\author{
E.A. Korznikova ${ }^{1, \dagger}$, S.Yu. Fomin ${ }^{2}$, S.V. Ustiuzhanina ${ }^{2}$, S.V. Dmitriev ${ }^{1}$ \\ †elena.a.korznikova@gmail.com \\ ${ }^{1}$ Institute for Metals Superplasticity Problems RAS, Khalturin St. 39, 450001 Ufa, Russia \\ ${ }^{2}$ Ufa State Aviation Technical University, K. Marx St. 12, 450000 Ufa, Russia
}

\begin{abstract}
Discrete breathers (DB) are spatially localized nonlinear vibrational modes in discrete lattices. The latest trend is the study of DB in various crystals. Many studies have been done recently for the two-dimensional (2D) model of crystal with Morse interatomic potentials. Two types of DB have been discussed for Morse crystals, the gap DB with soft type of nonlinearity and the hard-type anharmonicity DB with frequency above the phonon spectrum. Here we focus on the analysis of the effect of rigidity of the Morse potential on the properties of gap discrete breathers in the 2D biatomic crystal model of composition $\mathrm{A} 3 \mathrm{~B}$. Atoms $\mathrm{A}$ are ten times heavier than atoms $\mathrm{B}$ and this is the reason of the presence of a wide gap in the phonon spectrum of the crystal. The same Morse potentials are used to describe the A-A, B-B, and A-B interactions. Two of the three Morse potential parameters are fixed to unity without the loss of generality. For the third parameter, which defines the stiffness of the interatomic bond, two different values are considered. In both cases two polarizations of DB are investigated. It was found that for a smaller stiffness of the interatomic bond DB have larger maximal amplitude, regardless the type of polarization. On the other hand, they demonstrate a higher rate of radiation of the small-amplitude waves and thus, they have smaller lifetime.
\end{abstract}

Keywords: two-dimensional crystal, Morse potential, discrete breather.

\section{Introduction}

Nonlinear lattice dynamics has attracted a lot of attention in the last few decades in relation to the studies of the discrete breathers (DB), which can be defined as spatially localized nonlinear vibrational modes in defect-free lattices $[1,2]$. According to several recent experimental studies, DB can exist in various physical systems including optical, mechanical, electric, atomistic and other [2]. By now, a large volume of works has been performed to analyze properties of DB in various crystalline materials, particularly, the results of the molecular dynamics studies have been reported in [3-29]. Several works have been devoted to the study of DB in model crystals with Morse interatomic potentials [4-7], while other works to the study of DB in graphene and related materials [8-10]. In the work [8], for the first time, DB in a crystalline body has been studied with the help of DFT theory, using graphane as an example. DB in pure metals have been addressed in the works [11-12] and the discussion on how DB can affect physical properties of metals can be found in [13]. The works [14,15] discuss properties of DB in ionic crystals with $\mathrm{NaCl}$ structure. Many interesting studies have been reported on DB in ordered alloys [16-28].

DB can be divided into two wide classes by the type of their nonlinearity. In the case of soft (hard) nonlinearity, DB frequency decreases (increases) with increasing amplitude. A decrease of the frequency of the soft type nonlinearity DB with growth of its amplitude can result in entering the gap of phonon spectrum, if the latter exists. For the hard type nonlinearity modes the growing frequency can reach the values above the phonon spectrum (Fig.1).

It has been shown earlier that in the case of diatomic crystal with the mass ratio of light to heavy atoms less than 0.2 , the gap in the phonon spectrum is sufficiently wide and the gap DBs, having frequencies within the phonon gap, can be easily excited [3].

Until recentlyit wasbelieved that in realcrystalsinteratomic interactions tend to exhibit a soft type of nonlinearity, and

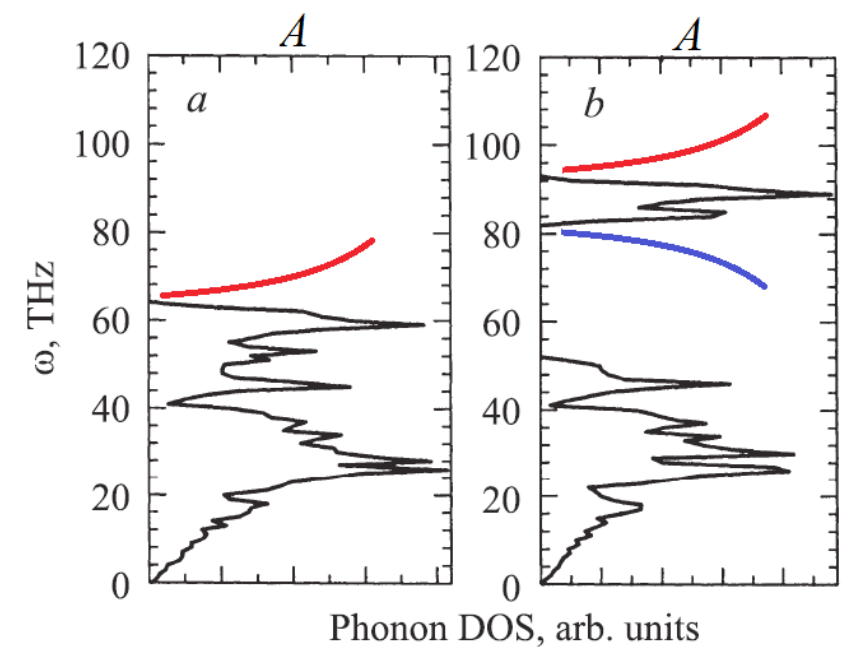

Fig. 1. Examples of phonon spectrum and hard type (red) and soft type (blue) DB for monoatomic (a) and biatomic (b) 2D Morse crystal. 
DB can only exist within a gap in the phonon spectrum of the crystal. However, in [23] a possibility of existence of DB with hard type of nonlinearity and with frequencies above the phonon spectrum was demonstrated by means of molecular dynamics modeling for $\mathrm{Ni}$ and $\mathrm{Nb}$. Later the possibility of the existence DB above the phonon spectrum of the crystal was confirmed in our works [4-6] for the case of two-dimensional (2D) monoatomic crystal with Morse interaction potential. This work was conducted in order to determine influence of potential parameters on characteristics of a soft type DB with frequency interval lying within the spectrum gap in the twodimensional (2D) diatomic Morse crystal.

\section{Simulation details}

We consider a model of 2D diatomic close-packed A3B type crystals with the interatomic distance $a$ mass with ratio of light to heavy components equal to 0.1 . Without loss of generality, the mass of atoms is considered to be unity. The interaction between atoms is described by the pairwise Morse potential:

$$
V(r)=D\left(e^{-2 \alpha\left(r-r_{m}\right)}-2 e^{-\alpha\left(r-r_{m}\right)}\right),
$$

where $r$ is the distance between a pair of atoms and, $D, \alpha, r_{\mathrm{m}}$ are the potential parameters. The function $U(r)$ has a minimum at $r=r_{\mathrm{m}}$, minimum depth (bond rupture energy) is $D$, and the parameter $\alpha$ determines the bond stiffness. Without loss of generality one can put $r_{\mathrm{m}}=1$ and $D=1$, selecting the appropriate unit of distance and energy. For the parameter $\alpha$ determining the rigidity of the interatomic bond the values $\alpha=4$ and $\alpha=5$ were considered. For the selected cut-off distance $7.5 \AA$, the equilibrium lattice parameter makes $\{0.9655659 ; 0.9881329\}$ for $\alpha=4$ and $\alpha=5$ correspondingly.

The potential has an inflection point, changing the sign of the second derivative from negative (on the right side) to positive one on the left. It is known that the second derivative defines the rigidity of interatomic bonds. Hence the Morse potential is a hard on small distances and soft at high (Fig. 2).

To excite a DB initial displacements of one light atom in a closely packed [110] atomic raw has been introduced with zero initial velocities of all atoms. We have investigated two cases of possible atomic shift directions, namely one parallel to [110] direction (fig 3, a) and one having $30^{\circ}$ polarization with [110] direction (fig 3, b) hereinafter referred as $0^{\circ}$ and $30^{\circ}$ polarization correspondingly. Both cases were studied with potential stiffness parameter $\alpha=4$ and $\alpha=5$.

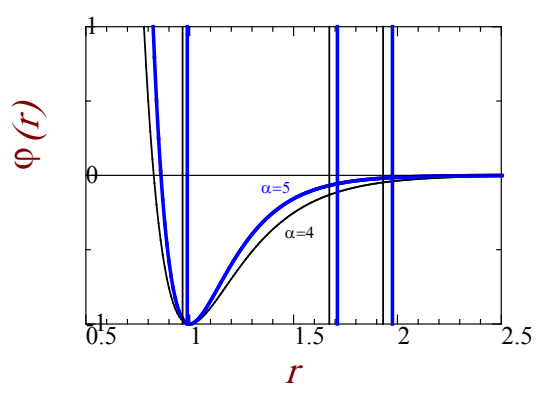

Fig. 2. Morse interatomic potentials for $\alpha=4$ and 5 .

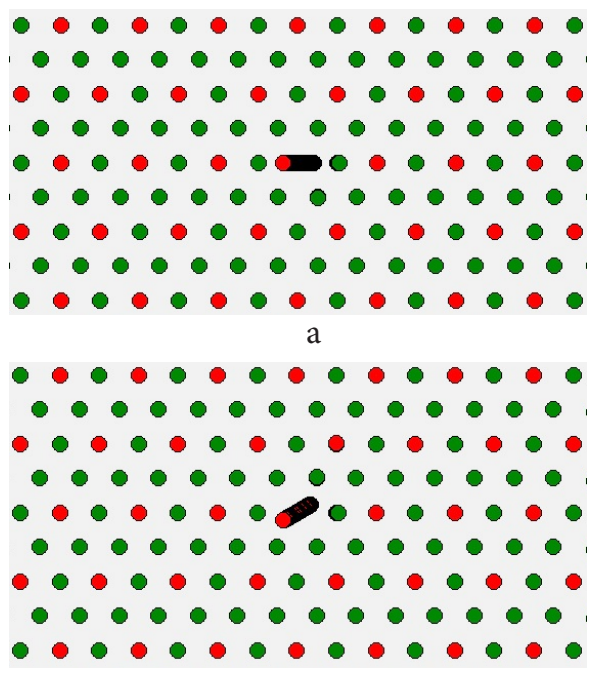

$\mathrm{b}$

Fig. 3. Stroboscopic image of atomic movement in a diatomic lattice with $0^{\circ}(\mathrm{a})$ and $30^{\circ}(\mathrm{b})$ polarization.

\section{Results and discussion}

Fig. 4 presents an example of atomic displacement within a $\mathrm{DB}$ in a Morse crystal with stiffness coefficient $\alpha=4$ exited in [110] direction with $0^{\circ}$ polarization. The amplitude of the DB makes $0.2 a$ and its variation reaches about $15 \%$ within the studied simulation period. The energy of the DB drops drastically in first 100 oscillation periods that correspond to transition stage of spreading the energy over the computational cell. The second stage of $E(t)$ dependence is characterized by slow linear decrease of energy with time.

It should be noted that this $\mathrm{DB}$ is immobile. It sits at one site and cannot move along the crystal lattice.

The phonon spectrum for $2 \mathrm{D}$ crystals with stiffness coefficient $\alpha=4$ and $\alpha=5$ are demonstrated on fig. 5. Increase

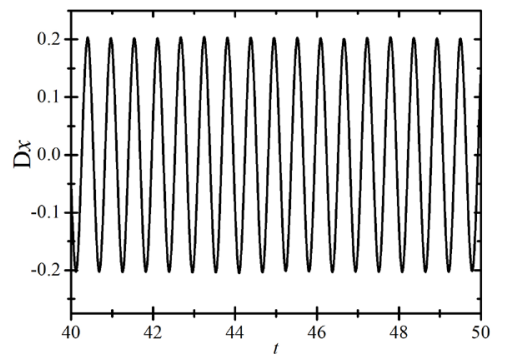

a

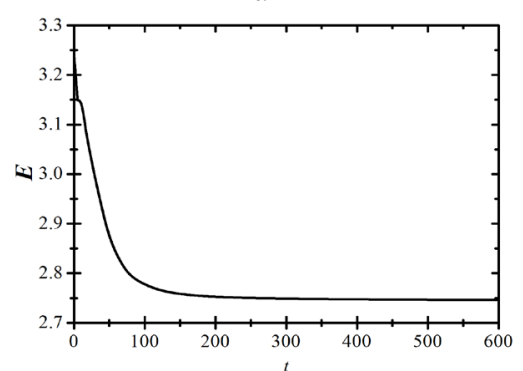

b

Fig. 4. An example atomic displacement as the functions of time stationary DB excited in a 2D Morse crystal $\alpha=4$ parallel to [110] direction (a) and dependence of DB energy on simulation time. 
of stiffness leads to growth of maximal phonon vibration frequencies and the gap width. A dependence of DB frequency on its amplitude for all investigated cases are shown on fig. 6. One can notice that change of DB polarization doesn't influence on DB properties. In case of $\alpha=4$ the DB amplitude varies from 0,16 to 0,32 a. For $\alpha=5$ the amplitude values are in the interval $(0,175-0,31)$ a. In all cases we have revealed the soft type nonlinearity with maximal vibration frequency below the value of the spectrum gap border shown by dashdot line on fig. 6.
We have performed the estimation of DB energy scattering velocity $\mathrm{dE} / \mathrm{dt}$ on the steady stage shown on fig. $4(\mathrm{~b})$ in the simulation time interval $200<\mathrm{t}<600$. The results of estimation are presented on fig. 7 and they reveal a monotonous decrease of DB scattering velocity for all investigated cases. One can mention that for $\mathrm{DB}$ with $30^{\circ}$ polarization demonstrate an imperceptibly lower $\mathrm{dE} / \mathrm{dt}$ values that could be explained by the fact that the direction of the oscillation of this DB has a lower density than that of [110] direction.

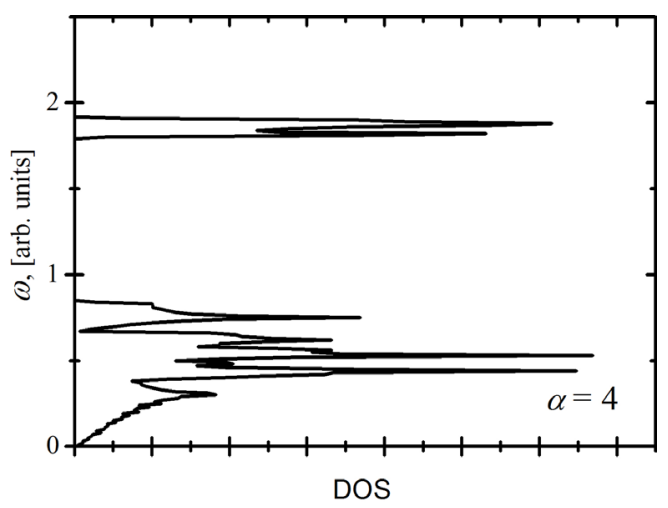

a

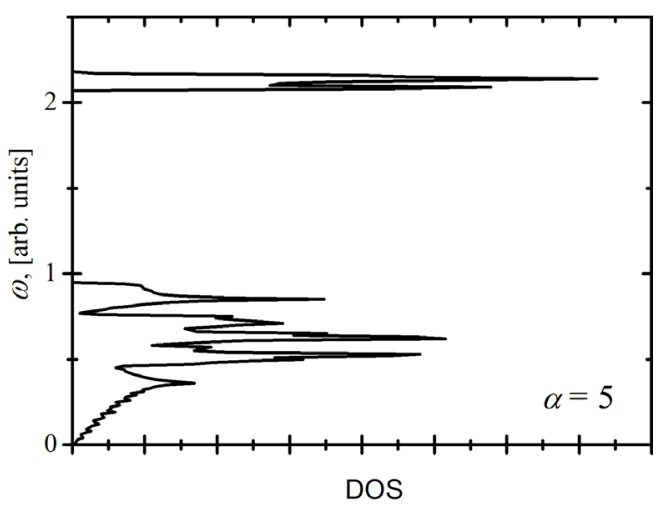

b

Fig. 5. Phonon spectrum of 2D diatomic Morse crystal for values of stiffness coefficient $\alpha=4$ (a) and $\alpha=5$ (b).

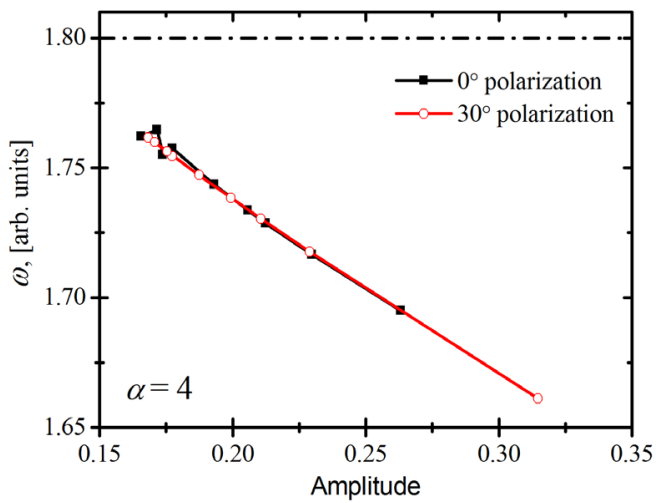

a

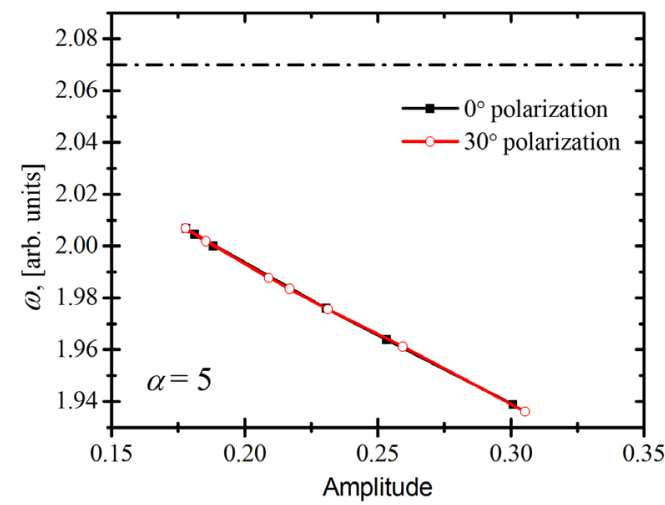

b

Fig. 6. Amplitude-frequency dependence of DB excited in 2D Morse crystals with $\alpha=4$ (a) and $\alpha=5$ (b) for cases of $0^{\circ}$ (black squares) and $30^{\circ}$ (open red circles) polarization. The dash-dot line shows the upper border of phonon spectrum gap.

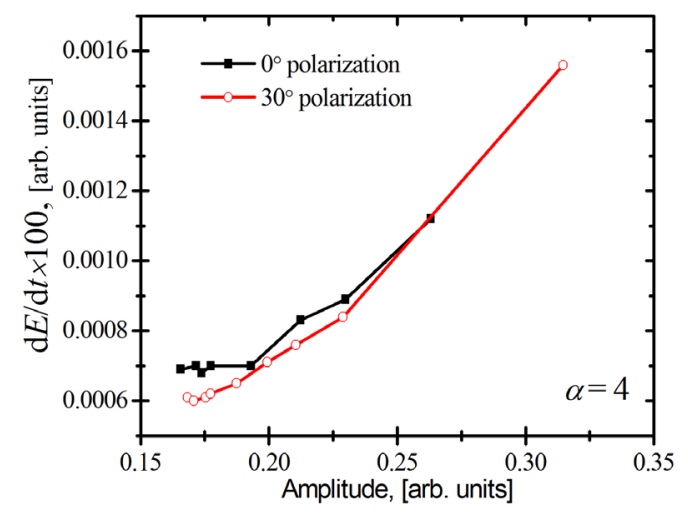

a

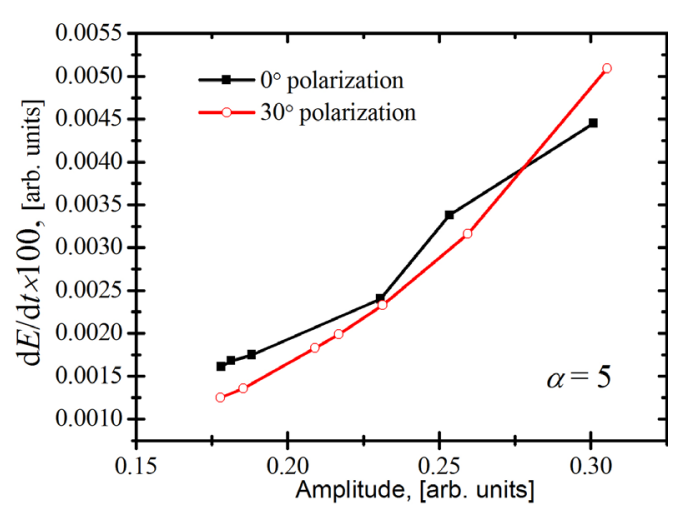

$\mathrm{b}$

Fig. 7. Energy scattering velocity $\mathrm{d} E / \mathrm{d} t$ for the DB excited in $2 \mathrm{D}$ Morse crystals with $\alpha=4$ (a) and $\alpha=5$ (b) for cases of $0^{\circ}$ (black squares) and $30^{\circ}$ (open red circles) polarization. 


\section{Conclusions}

In this work a study of properties of immobile DB localized on one atom in diatomic two-dimensional lattice with the Morse interaction for stiffness coefficient values $\alpha=4$ and $\alpha=5$ and $0^{\circ}$ and $30^{\circ}$ polarization has been performed.

It was revealed that frequency interval of DB existence is located within the phonon spectrum gap, its amplitude varies in the range $(0.15-0.325) \mathrm{a}$ and the $\mathrm{DB}$ is characterized by soft-type of non-linearity, i.e. decrease of amplitude results in growth of its oscillation frequency. The values of $\mathrm{dE} / \mathrm{dt}$, which characterizes the rate of $\mathrm{DB}$ energy radiation at the steady stage show a monotonic linear increase with growing DB amplitude. The results of the present study suggest that the investigated gap DB has very long lifetime and considerable amplitude, so that one can speculate that it can contribute to various physical events in diatomic crystals with a wide gap in the phonon spectrum.

Acknowledgements. The work of K.E.A. was supported by the RFBR grant 14-02-97029 r_povolzhe_a. S.V.D. thanks for the support the grant from the Russian Scientific Foundation \# 14-13-00982.

\section{References}

1. A.J. Sievers, S. Takeno. Phys. Rev. Lett. 61, 970-973 (1988).

2. S. Flach, A. Gorbach Phys. Rep. 467,1-116 (2008).

3. S.V. Dmitriev. Letters on Materials. 1(2), 78-83 (2011).

4. A.A. Kistanov. Fundamentalnie Problemi Sovremennogo Materialovedeniya. 11(1), 9-12 (2014).

5. A.A. Kistanov, E.A. Korznikova, S.Yu. Fomin, K. Zhou, S.V. Dmitriev. Letters on Materials. 4 (4), 315-318 (2014).

6. A.A. Kistanov, K. Zhou, E.A. Korznikova, S.Yu. Fomin, S.V. Dmitriev. Fundamentalnie Problemi Sovremennogo Materialovedeniya. 12(1), 103-107 (2015).

7. A.S. Semenov, E.A. Korznikova, S.V. Dmitriev. Letters on Materials. 5(1), 11-14(2015).

8. G.M. Chechin, S.V. Dmitriev, I.P. Lobzenko, D.S. Ryabov. Phys. Rev. B90, 045432 (2014).

9. E.A. Korznikova, J.A. Baimova, S.V. Dmitriev. Europhys. Lett. 102(6), 60004 (2013).

10. E.A. Korznikova, A.V. Savin, J.A. Baimova, S.V. Dmitriev. Pis'ma v zhurnal ehksperimental'noj i teoreticheskoj fiziki. 96 (3-4), 238-242 (2012).

11. A.A. Kistanov, A.S. Semenov, R.T. Murzaev, S.V. Dmitriev. Fundamentalnie Problemi Sovremennogo Materialovedeniya. 11(3), 322-325 (2014).
12. A.A. Kistanov, A.S. Semenov, R.T. Murzaev, S.V. Dmitriev. Fundamentalnie Problemi Sovremennogo Materialovedeniya. 11 (4-2), 572-577 (2014).

13. A.S. Semenov, R.T. Murzaev, A.A. Kistanov, J.V. Bebihov. Fundamentalnie Problemi Sovremennogo Materialovedeniya. 12 (1), 26-30 (2015).

14. V.I. Dubinko, A.V. Dubinko, S.V. Dmitriev. Letters on Materials. 3(3), 239-247 (2013).

15. A.A. Kistanov, S.V. Dmitriev. Phys. Solid State. 54(8), 1545-1548 (2012).

16. A.A. Kistanov, S.V. Dmitriev. Letters on Materials. 2(3), 143-146 (2012).

17. P.V. Zakharov, M.D. Starostepenkov, N.N. Medvedev, A.M. Eremin, A.V. Markidonov. Fundamentalnie Problemi Sovremennogo Materialovedeniya. 11(3), 388392 (2014).

18. P.V. Zakharov, M.D. Starostenkov, A.M. Eremin, A.V. Markidonov. Fundamentalnie Problemi Sovremennogo Materialovedeniya. 11(2), 260-265 (2014).

19. M.D. Starostenkov, P.V. Zakharov, N.N. Medvedev. Fundamentalnie Problemi Sovremennogo Materialovedeniya. 8(4), 40-44 (2011).

20. N.N. Medvedev, M.D. Starostenkov, P.V. Zakharov, O.V. Pozidaeva. Tech. Phys. Lett. 37 (3), 7-15 (2011).

21. N.N. Medvedev, M.D. Starostenkov, P.V. Zakharov, A.V. Markidonov. Letters on Materials. 3, 34-37 (2013).

22. N.N. Medvedev, M.D. Starostenkov. Russian Physics Journal. 55(11), 113-116 (2012).

23. N.N. Medvedev, M.D. Starostenkov and M.E. Manley. Journal of Applied Physics. 114, 213506 (2013).

24. M. Haas, V. Hizhnyakov, A. Shelkan, M. Klopov, A.J. Sievers. Phys. Rev. B84, 144303 (2011)

25. A.A. Kistanov, S.V. Dmitriev, A.P. Chetverikov, M.G. Velarde. Eur. Phys. J. B87, 211 (2014).

26. N.N. Medvedev, S.V. Dmitriev, M.D. Starostenkov. Fundamentalnie Problemi Sovremennogo Materialovedeniya. 4(3), 100-102 (2007).

27. O.V. Pozidaeva, S.V. Dmitriev, N.N. Medvedev, J.V. Bebihov, A.V. Samsonov, M.D. Starostenkov. Fundamentalnie Problemi Sovremennogo Materialovedeniya. 4(4), 102-107 (2007).

28. S.V. Dmitriev, N.N. Medvedev, R.R. Mulyukov, O.V. Pozidaeva, A.I. Potekaev, M.D. Starostenkov. Russian Physics Journal. 51(8), 73-79 (2008).

29. D.I.Bokij,E.A.Korznikova, S.V.Dmitriev. Fundamentalnie Problemi Sovremennogo Materialovedeniya 12(4), 403407 (2015). 